\title{
Experimental demonstration of a Brillouin optical frequency-domain reflectometry (BOFDR) sensor
}

\author{
R. Ruiz-Lombera*a, A. Minardo ${ }^{\text {b }}$, R.Berninic ${ }^{\mathrm{c}}$ J. Mirapeix $^{\mathrm{a}}$, J.M. Lopez-Higuera ${ }^{\mathrm{a}}$ L.Zeni $^{\mathrm{b}}$ \\ aPhotonics Engineering Group, Univ. of Cantabria, Ed. I+D Teleco, 39005, Santander (Spain) \\ ${ }^{b}$ Department of Industrial and Information Engineering, Second University of Naples, Via Roma 29, \\ 81031, Aversa (Italy) \\ 'Instituto per il Rilevamento Elettromagnetico dell'Ambiente, National Research Council, Via \\ Diocleziano 328, 81031, Naples (Italy)
}

\begin{abstract}
To measure the strain and/or temperature along an optical fiber with one-end access, a Brillouin optical frequency-domain reflectometry (BOFDR) technique is presented in this paper. It is based on detecting the spontaneous Brillouin scattering from a sinusoidally modulated pump light. Compared to the Brillouin optical frequency-domain analysis (BOFDA), this new BOFDR sensor approach presents the advantage that the measurements are free from the distorting components related to acoustic wave modulation, thus simplifying the associated data processing.
\end{abstract}

Keywords: BOFDR, spontaneous Brillouin scattering, Distributed optical fiber sensors

\section{INTRODUCTION}

Brillouin distributed optical fiber sensors are based on two different scattering processes, stimulated Brillouin scattering (SBS) and spontaneous Brillouin scattering (SpBS). These sensors operate based on the fact that the frequency of the Brillouin scattered light (the so-called Brillouin Frequency Shift (BFS)) depends on temperature and strain. Brillouin distributed sensors based on SpBS attract interest because of their capability to perform distributed strain and temperature measurements by accessing only one end of the fiber. Traditionally, there were two different approaches to perform measurements based on SpBS, the time and correlation domain techniques. The former is known as Brillouin Optical Time-Domain Reflectometry (BOTDR). In this method, the pump is a pulsed wave injected into the fiber, and the SpSB backscattered light is detected as a function of time ${ }^{1}$. In conventional BOTDR sensors, the spatial resolution is determined by the duration of the pulses and is typically limited to $1 \mathrm{~m}$. Shorter pulses are related to an improvement of the spatial resolution. However, the reduction of the pump pulse duration below the phonon lifetime involves a loss of accuracy in the BFS estimation given that the spectrum of the backscattered wave broadens rapidly ${ }^{2}$. In order to improve the spatial resolution, advanced configurations based for example on the differential method could be employed to reach sub-meter resolutions ${ }^{3}$. The other approach is based on the correlation domain and is known as Brillouin Optical Correlation-Domain Reflectometry (BOCDR). This sensor synthesizes a single correlation peak within the range of the fiber by modulating the frequency of the injected pump light ${ }^{4}$. The technique is capable of $\mathrm{cm}$-scale spatial resolution while keeping a narrowband Brillouin gain spectrum (BGS) and single-ended sensing capability given that the pump light is a continuous-wave (CW), but only a limited number of points could be measured.

In this paper, a novel reflectometry technique, here referred to as Brillouin Optical Frequency-Domain Reflectometry (BOFDR), is proposed. In analogy to its stimulated Brillouin scattering (SBS) counterpart (the Brillouin Optical Frequency-Domain Analysis (BOFDA)), it is based on the use of a pump light whose amplitude is sinusoidally modulated with a variable frequency. The corresponding modulation in the backscattered light is detected by use of a vector network analyzer (VNA). The spatial resolution and the sensing range of the sensor are determined by the frequency scan of the VNA employed to modulate the pump signal ${ }^{5}$.

*ruben.ruiz@unican.es; phone (+34) 942 206736; gif.teisa.unican.es

25th International Conference on Optical Fiber Sensors, edited by Youngjoo Chung, Wei Jin,

Byoungho Lee, John Canning, Kentaro Nakamura, Libo Yuan, Proc. of SPIE Vol. 10323,

103238L · () 2017 SPIE · CCC code: 0277-786X/17/\$18 · doi: 10.1117/12.2265733 
As the proposed technique is based on a frequency-domain configuration, some advantages (narrowband operation, averaging effect of inverse Fourier transform, reduced power levels ${ }^{5,6}$ ) appear compared with BOTDR sensors. But, this technique not only presents advantages with respect to BOTDR. We will also show that the proposed technique avoids the corruption of the acquired data observed in BOFDA sensors and caused by acoustic wave modulation ${ }^{7}$, thus simplifying the extraction of the BFS from the acquired BGS.

\section{PRINCIPLE OF OPERATION}

The nonlinear interaction between light launched and thermally excited acoustic phonons in an optical fiber provokes a scattered light due to spontaneous Brillouin scattering that undergoes a spectral shift equal to the BFS during the interaction and travels backward. This spectral shift is linearly dependent with temperature and strain along the fiber, allowing the extraction of these parameters from BFS measurements. The proposed BOFDR method works similar to the BOTDR, except from the spatial resolution that is achieved by impressing a sinusoidal modulation into the pump wave, rather than a pulsed modulation. The modulated backscattered wave, originating at each section of the fiber and propagating back to the fiber input due to SpBS, comprises both Stokes and anti-Stokes components. During the sweep of the modulation frequency, the amplitudes and phases of the detected Stokes powers are successively measured by the VNA. After data acquisition, the fiber response is obtained by a discrete inverse fast Fourier transform (IFFT), which provides the amplitude of the backscattered wave as a function of position. The modulation frequency scan determines the spatial resolution and the measuring range, according to 5 :

$$
\begin{gathered}
\delta z=\frac{c}{2 n} \frac{1}{f_{\max }-f_{\min }}, \\
L_{\text {max }}=\frac{c}{2 n} \frac{1}{\Delta f_{m}}
\end{gathered}
$$

where $f_{\min }$ and $f_{\max }$ denote the minimum and maximum modulation frequency, respectively, $\Delta f_{m}$ is the modulation frequency step, $n$ is the refractive index of the fiber and $c$ is speed of light in the vacuum. As in BOTDR, the information on local temperature and/or strain is achieved by spectrally analyzing the backscattered wave. As the latter is weak, its frequency is conveniently retrieved using a coherent detection scheme, as it will be described in the next section.

\section{RESULTS}

\subsection{Experimental set-up}

We show in Figure 1 the set-up used for the experiments. The light from an external cavity diode laser $(\lambda=1550 \mathrm{~nm}$, power $\sim 40 \mathrm{~mW}$, linewidth $\sim 100 \mathrm{kHz}$ ) is divided into the pump and reference light beams. The pump light is amplitudemodulated by an electro-optic modulator, biased at the half-power point and driven by the output of the VNA (Anritsu MS4630B). The modulated pump light is then amplified up to $5 \mathrm{dBm}$ and sent to a polarization scrambler operating at a rate of $700 \mathrm{kHz}$, before being injected into the sensing fiber. Through the optical circulators $\mathrm{C} 1$ and $\mathrm{C} 2$, the backscattered light is directed to a narrowband ( $\sim 5 \mathrm{GHz}$ ) fiber Bragg grating (FBG), with the aim of rejecting all the optical components apart from the Stokes wave of the SpBS. After optical filtering, the backscattered field is mixed to a fraction of the laser light acting as an optical local oscillator (amplified up to $8 \mathrm{dBm}$ ), producing a beat signal at the BFS frequency $(\sim 11 \mathrm{GHz})$. Then, the optical signal is detected with a $12-\mathrm{GHz}$ photodetector that provides the modulated signal to the heterodyne receiver. Finally, the resulting signal is sent to the VNA.

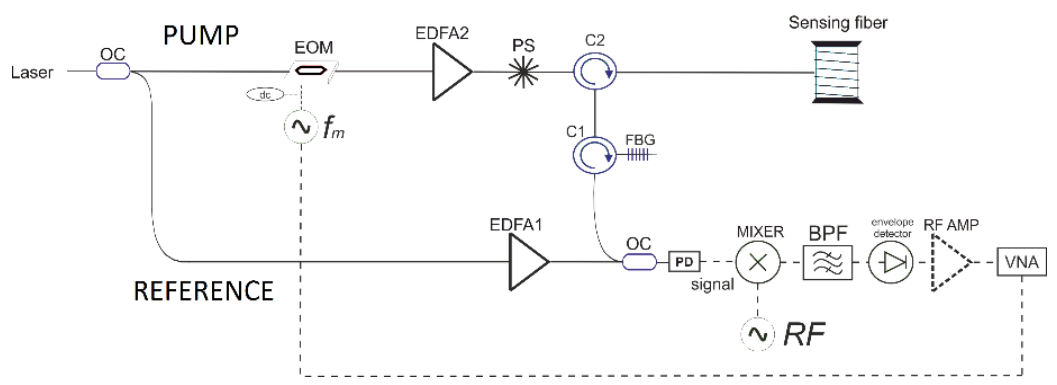

Figure 1. Experimental setup of BOFDR system. EOM: electro-optic modulator. OC: optical coupler; PS: polarization scrambler; PD: photodetector; BPF: electrical bandpass filter. 


\subsection{Experimental results}

To demonstrate the performance of BOFDR sensors, a distributed measurement has been carried out over a 100-m long fiber immersed into a hot water bath $\left(\mathrm{T} \sim 55^{\circ} \mathrm{C}\right)$, while leaving out of the bath two fiber segments of lengths $2 \mathrm{~m}$ and $1 \mathrm{~m}$, respectively. The VNA is operating at a resolution bandwidth (RBW) of $100 \mathrm{~Hz}$ and the modulation frequency $f_{m}$ ranging from $100 \mathrm{kHz}$ to $100 \mathrm{MHz}$, with $100 \mathrm{kHz}$ step. The choice of a maximum modulation frequency of $100 \mathrm{MHz}$, which corresponds to a spatial resolution of $\sim 1 \mathrm{~m}$, was dictated by the bandwidth of the bandpass filter in the heterodyne receiver stage. Moreover, an additional measurement under the same experimental conditions has been carried out using the BOFDA technique to compare.

We show in Figure 2 the two BFS profiles after applying first an IFFT to the frequency-domain data acquired and after applying a curve fitting to the resulting spectra. It can be observed that the BFS resolution of the two cold spots is more accurate when processing the BOFDR data, contrarily to the expected if we note that BOFDA measurements are characterized by a better signal-to-noise ratio (SNR). In the inset of Figure 2, it is possible to appreciate that the BGS acquired by BOFDR at the rightmost cold spot and in the middle of both is much wider than the BOFDA one, which is related to the SNR. It is also possible to see that the BGS acquired by the BOFDA system exhibits spurious peaks that introduce error in the BFS determination.

It is interesting to observe that the BGS linewidth of the BOFDR is $\sim 110 \mathrm{MHz}$, thus larger than the typical BGS linewidth in SpBS $(\sim 80 \mathrm{MHz})$. This fact must be attributed to the bandwidth of the electrical bandpass filter used in the heterodyne receiver.

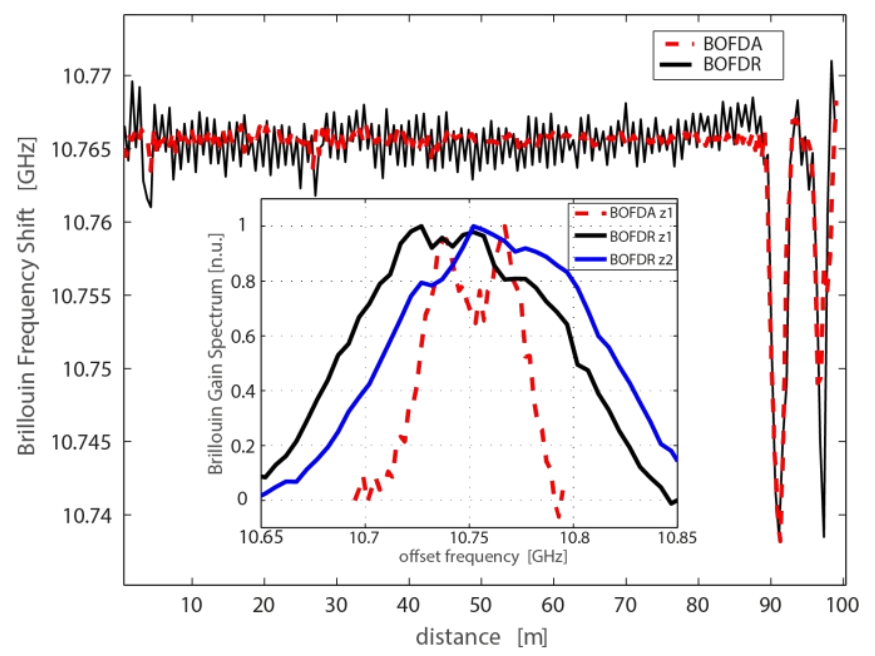

Figure 2. BFS reconstruction obtained by processing the BOFDA measurements or the BOFDR measurements. The inset shows the BGS acquired by the BOFDA at the position of the second cold spot $(\mathrm{z} 1=97 \mathrm{~m})$, and BGS acquired by the BOFDR at the same position ( $\mathrm{z} 1)$ and at an unerturbed position $(\mathrm{z} 2=94 \mathrm{~m})$.

From a theoretical point of view, it must be considered that in BOFDR the acoustic wave is thermally (rather than optically) generated $^{8}$; therefore, it may be argued that the acoustic wave is not modulated by the pump light as in BOFDA systems, with the consequence that no artifacts should be found in the acquired spectra.

A new measurement has been carried out in order to test that the proposed BOFDR method is free for the distorting features associated with the acoustic wave modulation that degrades the spatial resolution in BOFDA sensors. A distributed measurement over a $100 \mathrm{~m}$ long fiber at uniform temperature has been performed using BOFDR and BOFDA methods configured with the same optical power levels and the same modulation frequencies and RBW of the VNA. We compare in Figure 3 the normalized magnitude of the frequency-domain response of the 100-m fiber, acquired using the proposed BOFDR method (Fig. 3(a)) or a conventional BOFDA configuration (Fig. 3(b)). In the latter, it is appreciable the presence of three peaks in the frequency domain data when the modulation frequency is larger than the natural BGS linewidth, while the BOFDR data show no sub-peaks. The spurious peak is due to the interaction between the modulated component of the electrostrictively-driven acoustic wave and the pump wave ${ }^{7}$. This distorting features associated with the acoustic wave 
modulation are the reason of the degradation of the spatial resolution in BOFDA sensors. This explains why the BFS at the second cold spot in Figure 2 is erroneously resolved.
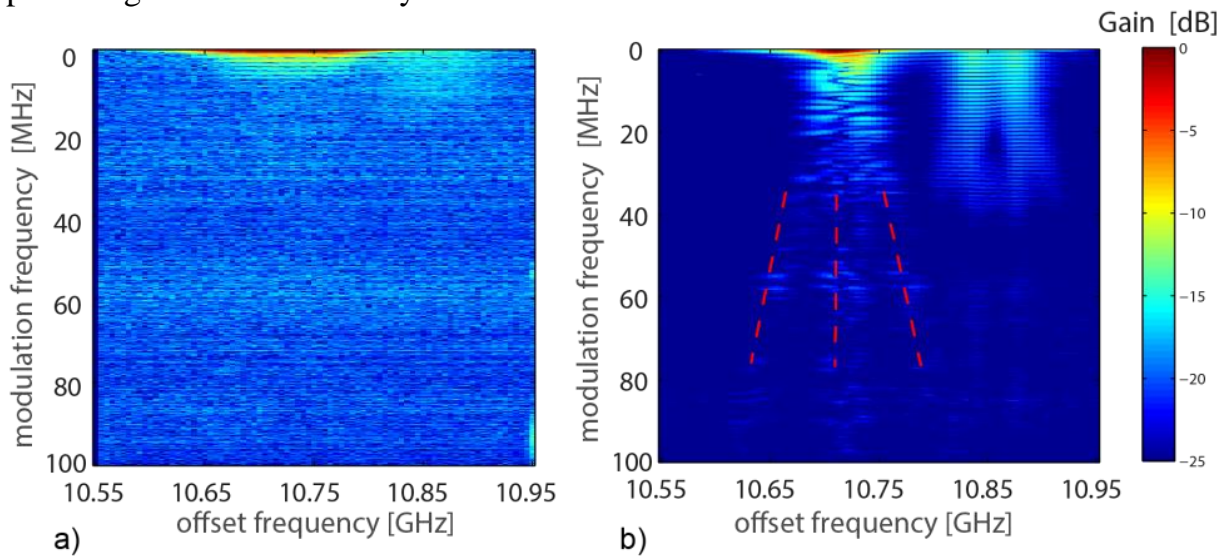

Figure 3. Frequency-domain data acquired using the proposed BOFDR method (a) or the BOFDA method (b). The red dashed lines in Fig. 3(b) highlight the three peaks in the frequency-domain Brillouin gain spectra. The gain visible at frequencies around $10860 \mathrm{MHz}$ is due to the SBS occurring along short pigtails spliced to the test fiber.

\section{CONCLUSIONS}

We have proposed a new Brillouin configuration based on the acquisition, in the frequency-domain, of the backscattered power caused by SpBS. By one-end access, distributed measurement of the BFS at a spatial resolution of $1 \mathrm{~m}$ over a fiber length of $100 \mathrm{~m}$ has been demonstrated. The commercial availability of compact, multi-GHz vector network analyzers may push the development of sub-meter BOFDR configurations, which could compete with conventional BOTDR systems.

\section{ACKNOWLEDGEMENTS}

This work has been supported by the projects MIUR-PON03PE_00155_1-OPTOFER and MIUR-PON03PE_00171_1GEOGRID of the Italian government, and by the project TEC2013-47264-C2-1-R and TEC2016-76021-C2-2-R of the Spanish government.

\section{REFERENCES}

[1] Kurashima, T., Horiguchi, T., Izumita, H., Furukawa, S.-i., and Koyamada, Y., "Brillouin optical-fiber time domain reflectometry," IEICE transactions on communications, 76(4), 382-390 (1993).

[2] Naruse, H., and Tateda, M., "Trade-off between the spatial and the frequency resolutions in measuring the power spectrum of the Brillouin backscattered light in an optical fiber," Applied optics, 38(31), 6516-6521 (1999).

[3] Li, Q., Gan, J., Wu, Y., Zhang, Z., Li, J., and Yang, Z., "High Spatial Resolution BOTDR Based on Differential Brillouin Spectrum Technique," IEEE Photonics Technology Letters, 28(14), 1493-1496 (2016).

[4] Mizuno, Y., Zou, W., He, Z., and Hotate, K., "Proposal of Brillouin optical correlation-domain reflectometry (BOCDR)," Optics express, 16(16), 12148-12153 (2008).

[5] Garus, D., Gogolla, T., Krebber, K., and Schliep, F., "Brillouin optical-fiber frequency-domain analysis for distributed temperature and strain measurements," Journal of lightwave technology, 15(4), 654-662 (1997).

[6] Farahani, M. A., and Gogolla, T., "Spontaneous Raman scattering in optical fibers with modulated probe light for distributed temperature Raman remote sensing," Journal of Lightwave Technology, 17(8), 1379 (1999).

[7] Bernini, R., Minardo, A., and Zeni, L., "Distributed sensing at centimeter-scale spatial resolution by BOFDA: measurements and signal processing,” IEEE Photonics Journal, 4(1), 48-56 (2012).

[8] Boyd, R. W., [Nonlinear Optics] Academic Press, (2008). 